\title{
Nuclear Structure Studies with Geanie at the LANSCE/WNR Facility
}

\author{
N. Fotiades, R. O. Nelson, M. Devlin, J. A. Becker, P. E. \\ Garrett, W. Younes, L. A. Bernstein and E. Tavukcu
}

This article was submitted to International Conference on the Labyrinth in Nuclear Structure, Crete, Greece 07/13/2003 - 07/19/2003

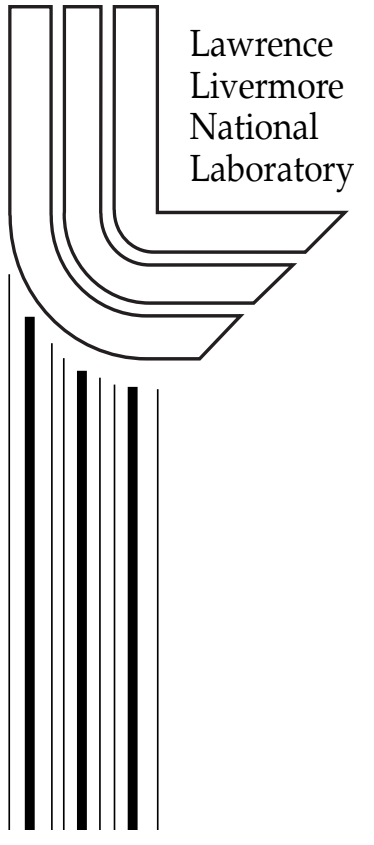

\section{August 1, 2003}


This document was prepared as an account of work sponsored by an agency of the United States Government. Neither the United States Government nor the University of California nor any of their employees, makes any warranty, express or implied, or assumes any legal liability or responsibility for the accuracy, completeness, or usefulness of any information, apparatus, product, or process disclosed, or represents that its use would not infringe privately owned rights. Reference herein to any specific commercial product, process, or service by trade name, trademark, manufacturer, or otherwise, does not necessarily constitute or imply its endorsement, recommendation, or favoring by the United States Government or the University of California. The views and opinions of authors expressed herein do not necessarily state or reflect those of the United States Government or the University of California, and shall not be used for advertising or product endorsement purposes.

This work was performed under the auspices of the U.S. Department of Energy by University of California, Lawrence Livermore National Laboratory under Contract W-7405-Eng-48. 


\title{
Nuclear structure studies with GEANIE at the LANSCE/WNR facility
}

\author{
N. Fotiades*, R. O. Nelson*, M. Devlin*, J. A. Becker ${ }^{\dagger}$, P. E. Garrett ${ }^{\dagger}$, W. \\ Younes $^{\dagger}$, L. A. Bernstein ${ }^{\dagger}$ and E. Tavukcu** \\ ${ }^{*}$ Los Alamos National Laboratory, Los Alamos, New Mexico 87545, USA \\ ${ }^{\dagger}$ Lawrence Livermore National Laboratory, Livermore, California 94550, USA \\ ${ }^{* *}$ North Carolina State University, Raleigh, North Carolina 27695, USA
}

\begin{abstract}
Recent results pertaining to nuclear structure from neutron-induced reactions on ${ }^{90} \mathrm{Zr}$, ${ }^{193} \mathrm{Ir},{ }^{196} \mathrm{Pt}$ and ${ }^{238} \mathrm{U}$ are presented. The data were taken using the GEANIE spectrometer comprised of 26 high-purity Ge detectors with 20 BGO escape-suppression shields. The broad-spectrum pulsed neutron source of the Los Alamos Neutron Science Center's WNR facility provided neutrons in the energy range from 0.6 to $200 \mathrm{MeV}$. The time-of-flight technique was used to determine the incident neutron energies. Results from shell model calculations for ${ }^{90} \mathrm{Zr}$ and from IBM-2 calculations for

${ }^{196} \mathrm{Pt}$ are generally in good agreement with the observed spectrum of excited states.
\end{abstract}

\section{INTRODUCTION}

The $\left(n, n^{\prime} \gamma\right)$ reaction at low neutron energies is a non-selective process. Hence, off-yrast states can be populated and studied in this type of reaction easier than in heavy-ion induced reactions.

The combination of a high-energy-resolution germanium-detector array (GEANIE) with the "white"-neutron source at the LANSCE/WNR facility enables the detection of $\gamma$-rays in neutron-induced reactions over a wide range of incident neutron energies. The prime aim of these experiments is to determine the partial cross sections for production of these $\gamma$-rays, but these experiments also provide additional results on the nuclear structure of the isotopes studied. Recent results on mainly off-yrast states of ${ }^{90} \mathrm{Zr},{ }^{193} \mathrm{Ir}$, ${ }^{196} \mathrm{Pt}$ and ${ }^{238} \mathrm{U}$ as studied in $\left(n, n^{\prime} \gamma\right)$ reactions with GEANIE are presented here.

\section{EXPERIMENTS}

Discrete $\gamma$-rays have been measured for isotopes populated in $(n, x n \gamma)$ reactions on ${ }^{90} \mathrm{Zr},{ }^{193} \mathrm{Ir},{ }^{196} \mathrm{Pt}$ and ${ }^{238} \mathrm{U}$ as a function of incident neutron energy in four separate experiments. The Ge $\gamma$-ray spectrometer GEANIE [1] (GErmanium Array for NeutronInduced Excitations) and neutrons from the "white" source neutron beam at the LANSCE/WNR (Los Alamos Neutron Science Center/Weapons Neutron Research) facility [2] were used.

GEANIE is comprised of 11 Compton-suppressed planar Ge detectors (Low Energy Photon Spectrometers - LEPS), 9 Compton-suppressed coaxial Ge detectors and 6 
unsuppressed coaxial Ge detectors. The energy of the neutrons was determined using the time-of-flight technique. The neutron flux on target was measured with a fission chamber [3], located upstream on the GEANIE neutron flight path. The fission chamber consists of two thin ${ }^{235} \mathrm{U}$ and ${ }^{238} \mathrm{U}$ foils and one uses the known ${ }^{235,238} \mathrm{U}(n, f)$ cross sections to deduce the neutron flux [3].

Two-parameter data were acquired for each detector consisting of time-of-flight relative to a fast proton-beam-pickoff signal and gamma-ray pulse height from the Ge detectors. Escape suppression was implemented by vetoing the associated Ge signals with the BGO signals in the hardware. Absolute detector efficiencies were determined using a variety of calibrated gamma-ray reference sources. Electronic "dead-times" were measured using scalers and corrections were applied to the data. During the experiments the data were stored on magnetic tapes for subsequent off-line analysis. Excitation functions for all the observed $\gamma$-rays were determined.

Symmetrized, two-dimensional matrices, constructed from the $\gamma-\gamma$ data, were used to investigate the coincidence relationships between the $\gamma$ rays. The placement of the newly observed $\gamma$ rays in the level schemes was based on the combination of the $\gamma$ $\gamma$ coincidence results with the deduced $\gamma$-ray excitation functions. The level-scheme systematics in neighboring isotopes was also used in some cases to further support the placement of these transitions.

As configured during the experiments described here, GEANIE covered too few unique detector angles (with respect to the neutron beam) to accurately determine angular distributions of the detected transitions. For ${ }^{90} \mathrm{Zr}$ [4] and ${ }^{196} \mathrm{Pt}$ [5] the angular distributions of the new transitions, necessary for spin and parity assignment to the new levels, were determined in separate $\left(n, n^{\prime} \gamma\right)$ experiments using the Ge-detector spectrometer and monoenergetic-accelerator-produced neutrons at the University of Kentucky van de Graaff accelerator facility.

\section{RESULTS AND DISCUSSION}

The results from each experiment are discussed briefly in the following four subsections:

$$
{ }^{238} \mathbf{U}\left(n, n^{\prime} \gamma\right){ }^{238} \mathbf{U}
$$

As an example of how easily an $\left(n, n^{\prime} \gamma\right)$ reaction can populate off-yrast states, a partial level scheme of ${ }^{238} \mathrm{U}$ is shown in Fig. 1 consisting of previously known transitions and levels [6] observed also in the present work. The absolute partial cross sections for the production of all transitions included in Fig. 1, as well as for several other transitions (a total of 45) from the ${ }^{238} \mathrm{U}(n, x n \gamma)(x \leq 4)$ reaction channels, were reported in Ref. [7].

The ground state band $2^{+}$state together with the first three off-yrast $2^{+}$states of ${ }^{238} \mathrm{U}$, forming part of the first and second $\beta$-vibrational bands and the $\gamma$-vibrational band, are shown in Fig. 1. Moreover, ten additional previously known off-yrast states with spins from 0 to $6 \hbar$ were observed [7]. 


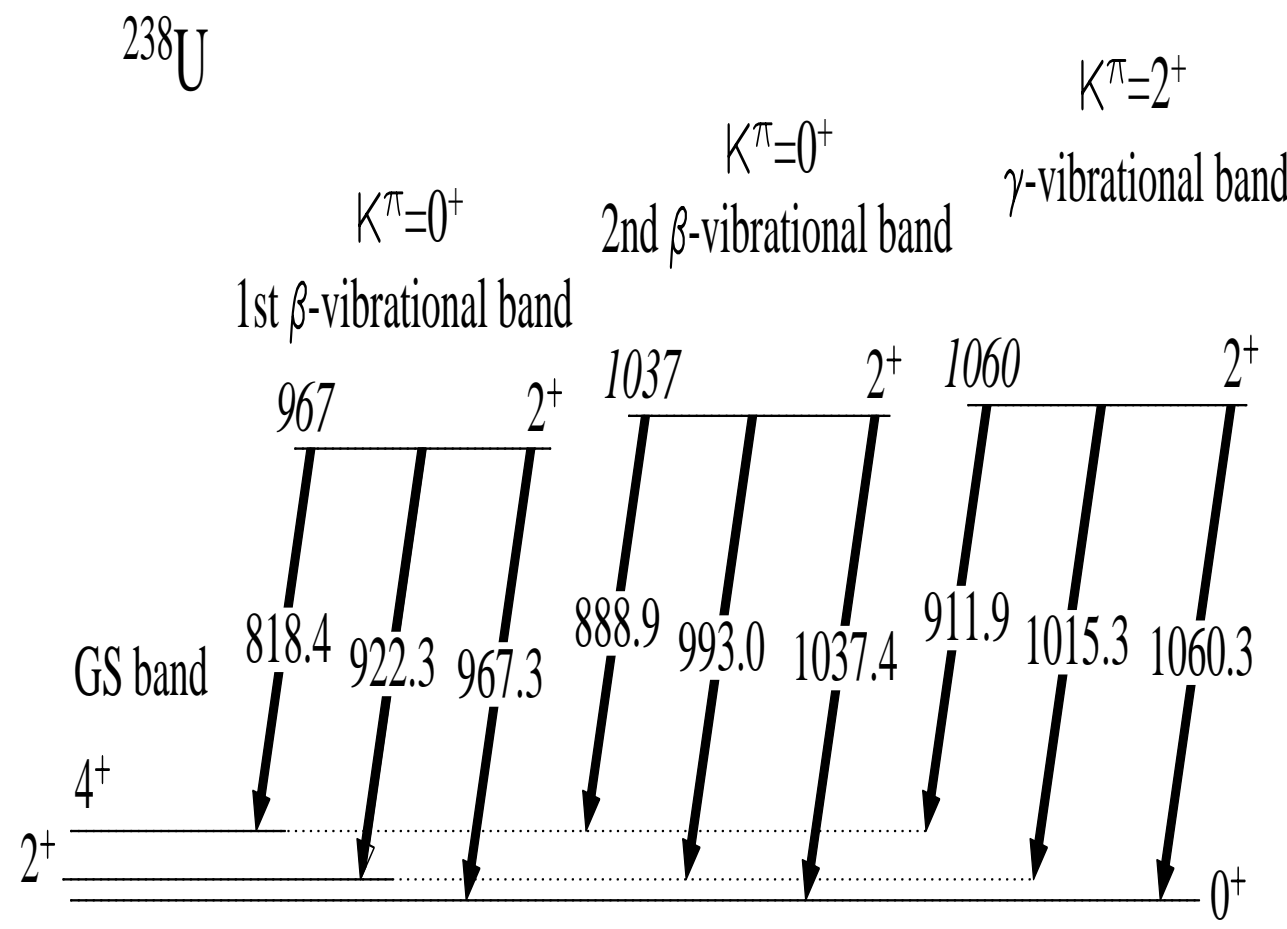

FIGURE 1. Previously known partial level scheme of ${ }^{238} \mathrm{U}$ with the first four $2^{+}$states of ${ }^{238} \mathrm{U}$ as observed in the GEANIE experiment. Transition and excitation energies are given in keV. The interpretation of the levels is also included.

A similar experiment has been recently performed with GEANIE with a ${ }^{232} \mathrm{Th}$ target [8] to study the vibrational bands in this isotope.

$$
{ }^{196} \mathbf{P t}\left(n, n^{\prime} \gamma\right){ }^{196} \mathbf{P t}
$$

Despite a great deal of previous work including $\left(n, n^{\prime} \gamma\right)$ studies, a total of 13 new levels, below $E_{x}=3 \mathrm{MeV}$, and 24 new transitions were assigned to ${ }^{196} \mathrm{Pt}$ in this experiment [5]. A partial level scheme of ${ }^{196} \mathrm{Pt}$ including two of the new levels and five of the new transitions is shown in Fig. 2. The absolute partial cross sections for the production of all transitions included in Fig. 2 are reported in Ref. [9].

With six bosons, ${ }^{196} \mathrm{Pt}$ has been shown [10] to be a good example of the $\mathrm{SO}(6)$ limit of the interacting boson model (IBM). The results of the IBM-2 (which distinguishes between proton and neutron bosons) calculations [5] for the two new states in Fig. 2 are in good agreement with the experimentally observed ones. 


\section{Experiment}

IBM-2

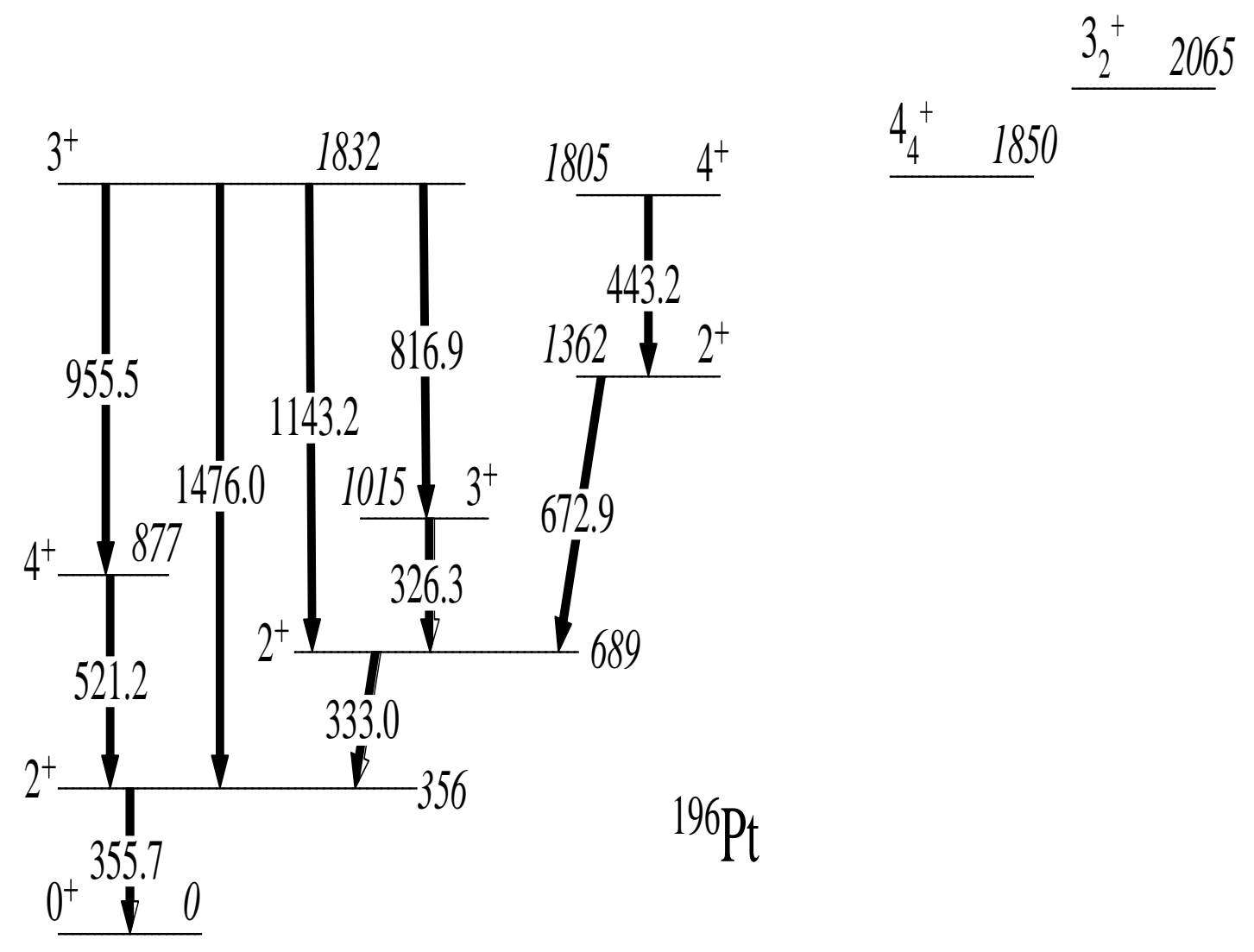

FIGURE 2. Partial level scheme of ${ }^{196} \mathrm{Pt}$ as deduced from the GEANIE experiment. Transition and excitation energies are given in keV. The 1805- and 1832-keV levels, as well as the transitions deexciting these levels, were observed for the first time. The results from IBM calculations for the new levels are also included.

$$
{ }^{193} \mathbf{I r}\left(n, n^{\prime} \gamma\right){ }^{193} \mathbf{I r}
$$

The data analysis of this experiment is still in progress. So far, a total of 7 new levels, below $E_{x}=1.5 \mathrm{MeV}$, and 10 new transitions have been assigned to ${ }^{193} \mathrm{Ir}$. A partial level scheme of ${ }^{193}$ Ir including two new levels and two new transitions is shown in Fig. 3 together with the previously known levels above the $80 \mathrm{keV}, 11 / 2^{-}, \tau=10.5$ days isomer [6]. The 19/2- spin and parity assignment of the newly observed $1025-\mathrm{keV}$ level is supported by the $\gamma-\gamma$ coincidences, the excitation function of the $546.8 \mathrm{keV}$ transition and the systematics of the corresponding 19/2 Ir isotopes [11]. The absolute partial cross sections for the production of all transitions 
included in Fig. 3 are reported in Ref. [11].

\section{${ }^{193}\left[\mathrm{I}\left[\left(n, n^{\prime}\right)^{193 m}{ }^{193}\right.\right.$}

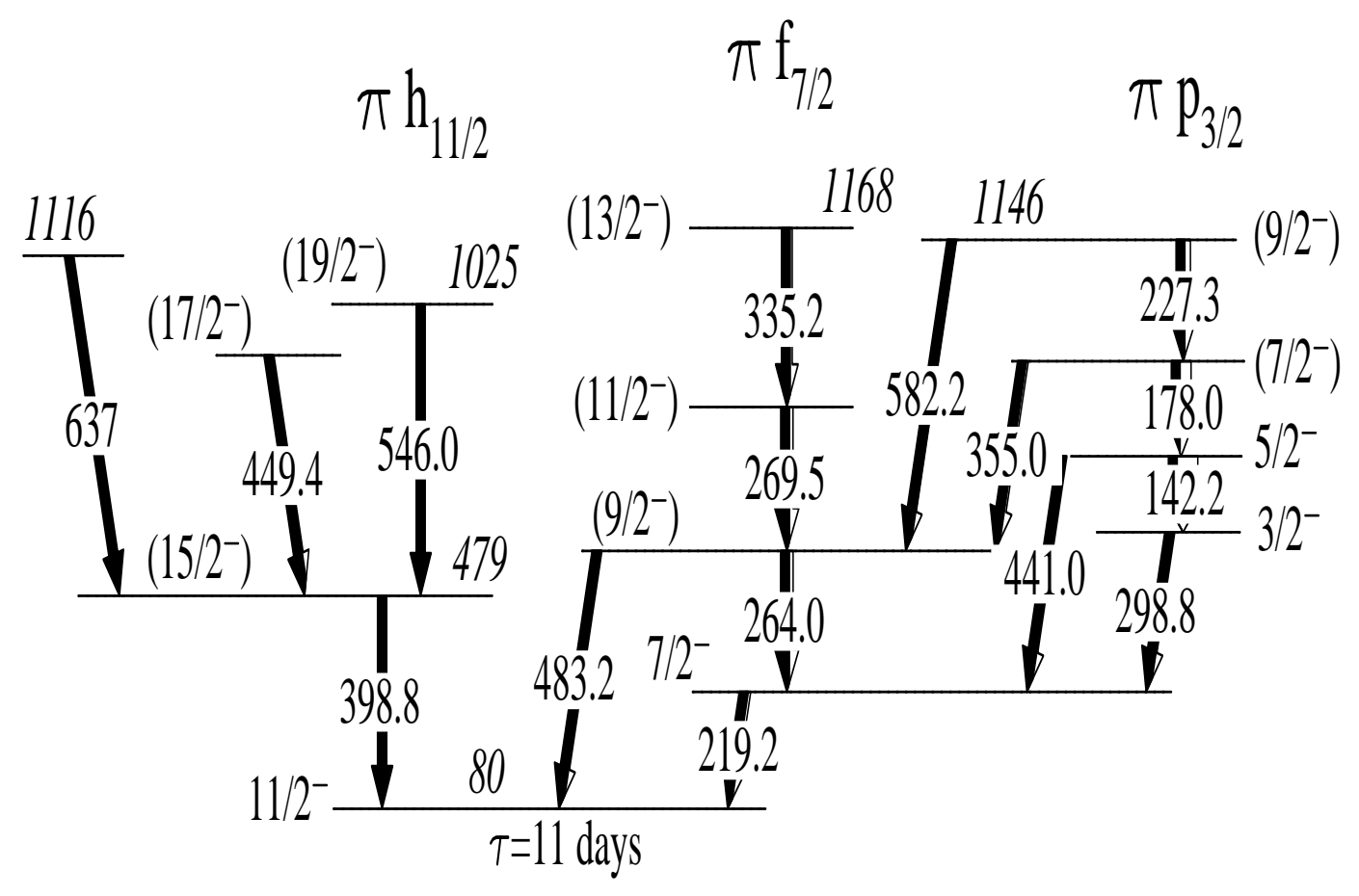

FIGURE 3. Partial level scheme of ${ }^{193} \mathrm{Ir}$, above the $80 \mathrm{keV}, 11 / 2^{-}, \tau=10.5$ days isomer, as observed in the GEANIE experiment. Transition and excitation energies are given in $\mathrm{keV}$. The $1025-$ and $1116-\mathrm{keV}$ levels, as well as the transitions deexciting these levels, are observed for the first time. The configuration of the bands is also given, where possible.

As an example of the quality of the $\gamma-\gamma$ data obtained with GEANIE, a spectrum obtained from a gate on the $398.8 \mathrm{keV}$ transition in Fig. 3 is shown in Fig. 4. As can be seen in Fig. 4, while counts are limited, generally, the gated spectra have greatly reduced backgrounds. Moreover, additional neutron energy gates can be introduced to further clean up the resulting spectra in cases where contamination is present.

An experiment will be performed with GEANIE in the near future with a ${ }^{197} \mathrm{Au}$ target [12] to establish the yet unknown structure above the $409 \mathrm{keV}, 11 / 2^{-}, \tau=8 \mathrm{sec}$ isomer [6] in this isotope, expected to be similar to the structure observed in Fig. 3. 


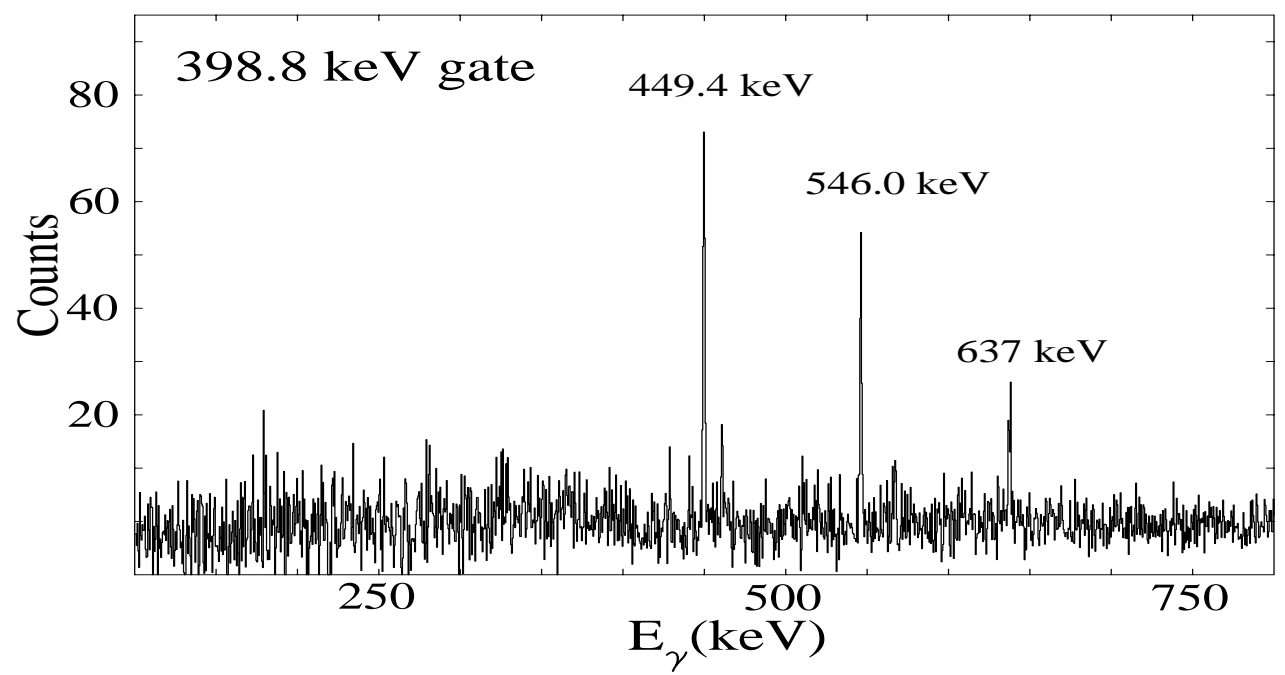

FIGURE 4. Spectrum in coincidence with the $398.8 \mathrm{keV}$ transition in Fig. 3 from the $\gamma-\gamma$ data. Since the spectrum is rather clean the use of an additional neutron energy gate has been deemed unnecessary.

$$
{ }^{90} \mathbf{Z r}\left(n, n^{\prime} \gamma\right){ }^{90} \mathbf{Z r}
$$

A total of 20 new levels, below $E_{x}=6 \mathrm{MeV}$, and 122 new transitions were assigned to ${ }^{90} \mathrm{Zr}$ in this experiment [4]. A partial level scheme of ${ }^{90} \mathrm{Zr}$ including two of the new levels and seven of the new transitions is shown in Fig. 5. The absolute partial cross sections for the production of all transitions included in Fig. 5 are reported in Ref. [13].

Shell model calculations, assuming a ${ }^{88} \mathrm{Sr}$ closed core and employing a space spanning twelve protons in the $0 f_{5 / 2}, 1 p_{5 / 2}, 1 p_{1 / 2}$, and $0 g_{9 / 2}$ orbits, reproduce the spectrum of excited states very well [4], as can be seen, for instance, for the newly observed $3^{+}$ state in Fig. 5. In the case of the $5^{-}$state in Fig. 5, the calculation predicts three different $5^{-}$states in this excitation energy region, so, it is not clear which one (or, possibly, which combination of these states) can be used for the interpretation of the observed state.

\section{SUMMARY}

In conclusion, off-yrast states were studied in ${ }^{90} \mathrm{Zr},{ }^{193} \mathrm{Ir},{ }^{196} \mathrm{Pt}$ and ${ }^{238} \mathrm{U}$ using neutroninduced reactions and $\gamma$-ray spectroscopy. The pulsed neutron beam from the LANSCE/WNR facility, combined with the GEANIE spectrometer, is a unique and powerful facility for such studies. Several new $\gamma$ rays were observed and were placed in the level schemes using both the deduced $\gamma$-ray excitation functions and the $\gamma-\gamma$ coincidence results. Calculations, using a shell model for ${ }^{90} \mathrm{Zr}$, and IBM-2 for ${ }^{196} \mathrm{Pt}$, are generally in good agreement with the observed excitation energy spectra. 


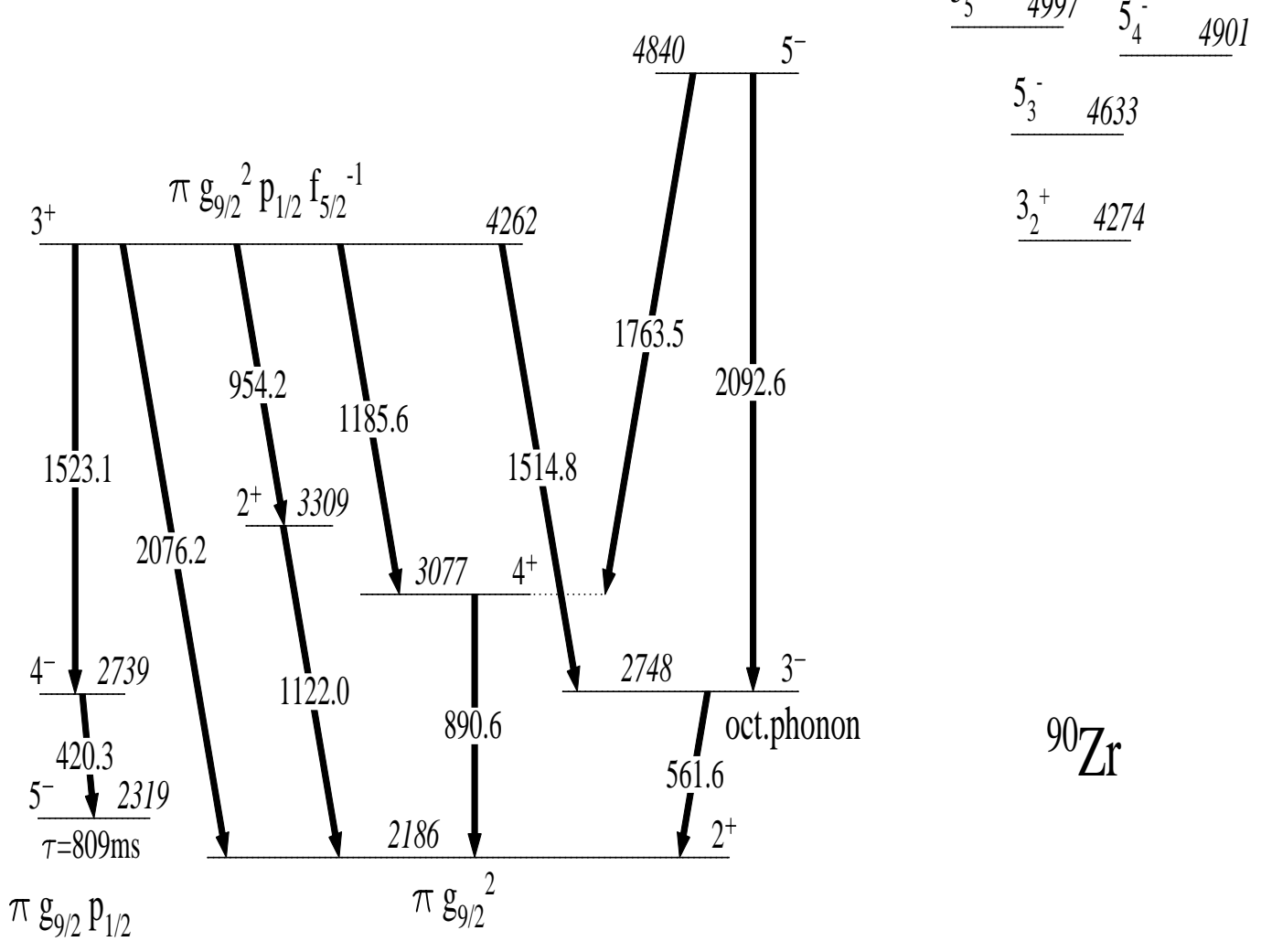

FIGURE 5. Partial level scheme of ${ }^{90} \mathrm{Zr}$ as deduced from the GEANIE experiment. Transition and excitation energies are given in keV. The 4262- and 4840-keV levels, as well as the transitions deexciting these levels, were observed for the first time. The results from shell model calculations for the new levels are also included.

\section{ACKNOWLEDGMENTS}

This work was performed under the auspices of the U.S. Department of Energy by the University of California, Los Alamos National Laboratory under contract no. W7405-ENG-36 and Lawrence Livermore National Laboratory under contract no. W7405-ENG-48, and benefitted from the use of the Los Alamos Neutron Science Center supported under contract no. W-7405-ENG-36.

\section{REFERENCES}

1. J. A. Becker, R. O. Nelson, Nuclear Physics News International 7, 11 (1997).

2. P. W. Lisowski, C. D. Bowman, G. J. Russell, S. A. Wender, Nucl. Sci. Eng. 106, 208 (1990). 
3. S. A. Wender, S. Balestrini, A. Brown, R. C. Haight, C. M. Laymon, T. M. Lee, P. W. Lisowski, W. McCorkle, R. O. Nelson, W. Parker, Nucl. Instr. Meth. A 336, 226 (1993).

4. P. E. Garrett, L. A. Bernstein, J. A. Becker, W. Younes, E. M. Baum, D. P. DiPrete, R. A. Gatenby, E. L. Johnson, C. A. McGrath, S. W. Yates, M. Devlin, N. Fotiades, R. O. Nelson, B. A. Brown, Phys. Rev. C 62, in press (2003).

5. E. Tavukcu, L. A. Bernstein, K. Hauschild, J. A. Becker, P. E. Garrett, C. A. McGrath, D. P. McNabb, W. Younes, P. Navratil, R. O. Nelson, G. D. Johns, G. E. Mitchell, and J. A. Cizewski Phys. Rev. C 65, 064309 (2002).

6. R. B. Firestone, V. S. Shirley, C. M. Baglin, S. Y. Frank Chu, and J. Zipkin, Table of Isotopes, Wiley, New York (1996)

7. N. Fotiades, G. D. Johns, R. O. Nelson, M. B. Chadwick, M. Devlin, W. S. Wilburn, P. G. Young, D. E. Archer, J. A. Becker, L. A. Bernstein, P. E. Garrett, C. A. McGrath, D. P. McNabb, W. Younes, submitted to Phys. Rev. C (2003).

8. P. E. Garrett, W. Younes et al., LLNL report, to be submitted.

9. E. Tavukcu, L. A. Bernstein, K. Hauschild, J. A. Becker, P. E. Garrett, C. A. McGrath, D. P. McNabb, W. Younes, M. B. Chadwick, R. O. Nelson, G. D. Johns and G. E. Mitchell, Phys. Rev. C 64, 054614 (2001).

10. J. A. Cizewski, R. F. Casten, G. J. Smith, M. L. Stelts, W. R. Kane, H. G. Börner, W. F. Davidson, Phys. Rev. Lett. 40, 167 (1978).

11. N. Fotiades, R. O. Nelson, M. Devlin, M. B. Chadwick, P. Talou, J. A. Becker, L. A. Bernstein, P. E. Garrett, D. P. McNabb, W. Younes, LANL report, to be submitted (2003).

12. N. Fotiades, R. O. Nelson, M. Devlin, J. A. Becker, L. A. Bernstein, W. Younes, P. E. Garrett, LANL proposal No 2003503, (2003).

13. P E Garrett, J A Becker, L A Bernstein, W E Ormand, W. Younes, R O Nelson, M Devlin, N Fotiades, Bull. Am. Phys. Soc. 47, No 2, 161 (2002), and P. E. Garrett et al., LLNL report, to be submitted. 